\title{
PRACTICAL DESIGN AND IMPLEMENTATION OF A CAVE SYSTEM High Quality Four Walls CAVE howto
}

\author{
Achille Peternier, Sylvain Cardin \\ Virtual Reality Laboratory (VRLab), Ecole Polytechnique Fédérale de Lausanne (EPFL), Lausanne, Switzerland \\ achille.peternier@epfl.ch, sylvain.cardin@epfl.ch \\ Frédéric Vexo, Daniel Thalmann \\ Virtual Reality Laboratory (VRLab), Ecole Polytechnique Fédérale de Lausanne (EPFL), Lausanne, Switzerland \\ frederic.vexo@epfl.ch,daniel.thalmann@epfl.ch
}

Keywords: CAVE, low cost, immersive Virtual Reality, calibration, tracking, stereographic rendering.

\begin{abstract}
CAVE systems are nowadays one of the best Virtual Reality (VR) immersive devices available for rendering virtual environments. Unfortunately, such kind of hardware is extremely expensive, complex and cumbersome, thus limited in its spread. Several cheaper solutions already exist, but they implement usually only a subset of features of a professional CAVE. In this paper we describe how we have built a low cost CAVE with four screens (three walls and a floor), stereographic rendering and user tracking by only using hardware commonly available on the market and free software, we show the different solutions and work-around we implemented to solve the problems we encountered and we conclude with an evaluation of our system by using two applications we developed with it.
\end{abstract}

\section{INTRODUCTION}

Immersive environments giving the illusion of being surrounded by a fictive world are a key feature required by many Virtual Reality applications and are extremely difficult to simulate. Visual immersion needs specific, expensive and cumbersome hardware, such as head-worn displays (HMDs), large displays or CAVE systems (Sutcliffe et al., 2006). HMDs offer a good level of immersion, but often suffer of a small field of view and isolate the user and his/her body both from the real and the virtual world (Czernuszenko et al., 1997). Spatially Immersive Displays (SIDs), like wall-displays and CAVEs, have the advantage of being multi-user, to allow persons to be physically within the virtual environment and feature a wide field of view. Many studies (Tyndiuk et al., 2005) (Buxton et Fitzmaurice, 1998) showed that devices based on large displays offer a better immersion and cognitive interaction with a virtual 3D environment. Due to the high cost of professional solutions and their complexity, the proliferation of such kind of environments is limited to institutes or organizations able to pay and manage such structures.
In this paper we describe how we have built a four sides CAVE (three walls, one floor) by using standard market products and internally developed software, without requiring any third part or high-end professional product. Despite of this, we achieved to manage a very flexible, robust, high quality and fast CAVE environment, featuring stereographic rendering, a good calibration system for walls and sensors, head-tracking and last generation graphics comparable to most recent video games. We expose how we managed every aspect and solved all the accuracy and practical problems of this project in order to show how home-made SIDs can be created with a minimal effort and in a relatively cheap way, without sacrificing the quality. We compare our results with other similar framework and establish a fair evaluation. Finally, we illustrate some applications we developed with our system to show what it is possible to handle with our environment.

\section{RELATED WORK}

The CAVE Automatic Virtual Environment (or simply CAVE) has been originally conceived in 1992 
by Thomas Defanti and Dan Sandin and implemented by Carolina Cruz-Neira at the University of Illinois at Chicago (Cruz-Neira et al., 1992). The idea behind this project was to create a VR system without the common limitations of previous VR solutions, like poor image resolution, inability to share the experience directly with other users and the isolation from the real world. A head tracking system is used to produce the correct stereo perspective: this allows the user to see his/her entire environment from the correct viewpoint, thus creating a compelling illusion of reality. Real and virtual objects are blended in the same space and the user can see his/her body interacting with the environment.

Over the past decade, interest and development of CAVEs and SIDs have become increasingly significant, some of them becoming also commercial products like Barco (www.barco.com) and VRCO (www.vrco.com). Despite of the amount of development in this direction, both professional and customized solutions are still extremely expensive, because they are often close to prototypes requiring specific cares, like the CAVE-like display developed by Gross et al. (Gross et al., 2003) to project and acquire 3D video content.

There also exist reduced versions of CAVE systems, with fewer walls or even transportable. One of them is the V-CAVE (made with only two walls, hence the name V-CAVE, because the two screens form a letter "vee"). Two digital projectors point into the corner of a room, avoiding the requirement of dedicated screens (Jacobson, 2003). This system (Jacobson et Hwang, 2002) is also based on the top of a game engine which offers good quality graphics on personal computers but sacrifices the versatility of their approach for context other than a walkthrough of static pre-processed models, thus reducing the use of their software in contexts with extremely dynamic graphics. We aimed at a more complex system with four walls and a more generic engine in order to be used in contexts also requiring extremely dynamic geometries or modifications to the scene graph.

Different approaches have been studied for tracking the user position inside the CAVE. The recurrent problematic depends on the physical characteristics of the system. The most used indoor tracking systems are based on active vision algorithms or on magnetic tracking. Both of these solutions are costly and have specific drawbacks. The vision based approach with markers, like the Vicon system, uses video acquisition from cameras and is sensible to obstructions. In a CAVE framework, the field of view of the cameras has to be wide enough to cover a large area of interaction, thus multiple cameras are necessary to improve tracking accuracy and in order to cover a larger area. There also exist cheaper implementations using passive vision based systems with passive markers and standard cameras, running on free library such as ARtoolkit and good enough to produce accurate results in stereo acquisition (Koo et al, 2004).

Magnetic tracking, using systems such as the MotionStar $^{\text {tm }}$ one (www.ascensiontech.com), is widely used for full body motion tracking. The main disadvantage in using this technology is that most of the CAVEs are built on iron frames and metallic masses may alter the magnetic field measured by the sensors. Research at the Illinois University ( $M$. Ghazisaedy et al., 1995) offers a good illustration of the magnetic field distortions. They also present a method to calibrate the system to improve the accuracy, by correlating the measurements with ultrasonic sensors.

Sauter described a low-cost CAVE solution based on generic Windows and Macintosh computers to make this technology more accessible (Sauter P. M., 2003). We adopted a similar but updated architecture for our framework. We also extended the low-cost idea to the tracking system and by improving calibration tools to solve common problems of home-made virtual devices. Based on these experiences, the next part will describe our system and its different implementations.

\section{SYSTEM}

In this section we describe our solution, first with a brief overview of the whole thing then by explaining every aspect in detail.

\subsection{System overview}

Our CAVE features three walls and a floor. We used eight off the shelf LCD beamers (Canon LV-7210) connected to four personal computers (Intel Dual Xeon $3 \mathrm{GHz}$, with dual DVI output NVidia Geforce 7800 ), generating and projecting images on a cinema screen folded in the form of a box (for the walls) and on a white wooden panel (on the ground).

Back-projection has been used for the walls, direct one for the floor. A fifth master PC leads the four clients, through a server-client architecture connected via a private 1 Gigabit LAN. The server PC also manages a 5.1 audio system to bring spatial sounds in the environment.

The system works both in mono and stereographic rendering, either active (shutter glasses) or passive (red and blue glasses). The CAVE is 2.20 meters high, 2.50 meters large and 1.80 meters depth. Up to three users can comfortably stay within the framework. 
Software side, our environment uses a graphic engine specifically developed in our lab: server and clients run local instances of the same engine synchronized through the server. Synchronization includes models, scene graph, textures, shaders, etc. thus obtaining a full dynamic environment.

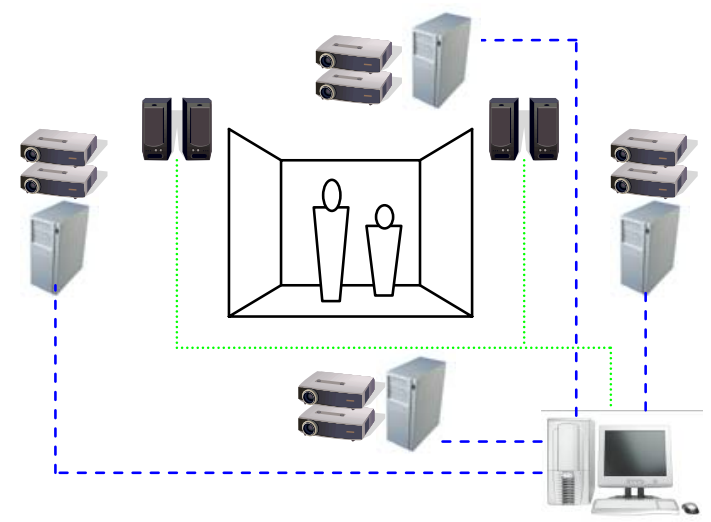

Figure 1: hardware setup overview.

\subsection{Graphic and audio system}

Our CAVE is running a modified version of an internally developed graphic engine first created for pedagogical purposes and called MVisio (Peternier, 2006).

MVisio (Mental Vision) is a lightweight, robust and user-friendly 2D/3D graphic engine. The goals of MVisio are several: offering an extremely easy to use and intuitive interface to 2D/3D graphics, being able to run on almost every available desktop PC or laptop by automatically adapting the rendering quality and settings, being also able to work on heterogeneous devices (PC, PocketPC) or operative systems (Windows and Linux), being compact in sizes and system requirements and finally being fast and modern (featuring advanced rendering techniques such as soft shadows and post-processing effects like bloom lighting through OpenGL and OpenGL|ES). Because of the flexibility and already tested robustness of this software, we decided to add CAVE rendering to its features.

MVisio for CAVE uses a server-client architecture based on low-level TCP/IP communication between wall and floor client PCs and the main server computer. Thanks to the speed of the local Gigabit LAN the latency generated by the network is extremely low. Client machines run on a minimal installation of Windows XP. A service runs perpetually waiting for connections from an MVisio for CAVE server. MVisio server sends a wake-up re- quest to all the services running on the different client machines, the service starts an MVisio local client which is an interpreter of high-level commands (move, rotate, display, etc.) sent from the server. Every operation effectuated on the server PC is forwarded to the clients. This include data loading (textures and geometries are synchronized at startup), shaders, modifications to the scene-graph, etc. Running MVisio on a local PC or in the CAVE just requires for the end-user to modify a couple of lines of code during initialization of the graphic engine: everything else is automatically handled by our software. This task can also be skipped by using configuration files, in order to allow to exactly the same source code to switch from the single PC version to the CAVE version of MVisio in a completely transparent way, by just passing a different configuration file as argument. MVisio CAVE clients can be considered as copies of the MVisio engine running remotely and directly manipulated by the user: information is synchronized through the network connectivity between the machines. The different frusta are computed server side and independently forwarded to the specific clients. The architecture is completely versatile and can handle from one remote client up to any arbitrary number of displays. It is interesting to mention that our architecture can also be used as remote rendering system to display real-time images on a remote PC somewhere in the world and not being a part of the CAVE private network. This feature also theoretically allows data synchronization between two or more remote CAVEs.

The audio engine has also been developed in our laboratory. It features spatial audio positioning through the OpenAL API (www.openal.org), audio streaming (from .WAV and .OGG files) as well as DSP effects through Creative EAX 2.0 (developer.creative.com). The main advantage of using our own audio engine is that it perfectly fits with MVisio, so adding audio sources to a scene graph is straightforward and robust. Contrarily to the graphic subsystem, the audio engine doesn't require to be instanced on each CAVE client and runs only on the server PC that is connected to the five Dolby 5.1 loudspeakers (see figure 1). Audio and images are synchronized by using the server timer.

\subsection{CAVE display calibration}

We used a market level flat home-cinema screen as display for the three walls and a white painted wooden panel as floor. To fold the home-cinema screen in order to create a box surrounding the user we used a transparent nylon wire stretched along the corners of an iron frame. This solution caused a ma- 
jor drawback: the screen folded with a curve around the corners, assuming the shape of a parenthesis. Also projecting images from two separate projectors generates an unavoidable misalignment, despite an accurate calibration of their convergence.

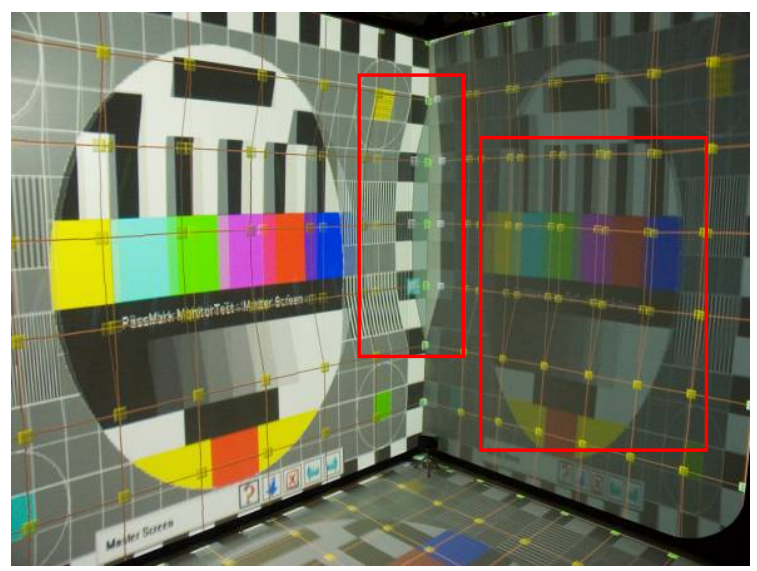

Figure 2: Non calibrated walls, notice the glitch between the two displays (left red box) and the grid not aligned for stereographic rendering (right red box).

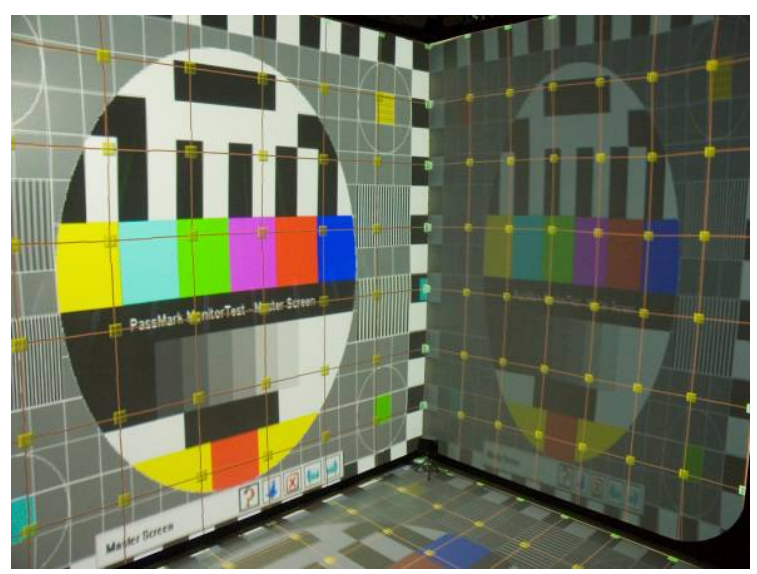

Figure 3: Walls after calibration.

We managed to solve these problems by meanings of calibration software, allowing the user to manually draw a shape accurately following the screen borders and by using this shape as a polygon to render the images into. Basically, the CAVE performs a high resolution (1024x1024 pixels) renderto-texture using the OpenGL frame-buffer object extension. This texture is then mapped onto the polygon previously adapted to fit the display shape. To improve image superposition for stereo rendering, we also used a grid with moveable control points. By matching the control points of the grids projected from the two projectors, we avoided deformations caused by beamers being physically pro- jecting from two points slightly different in the space. An accurate calibration showed per-pixel accuracy and almost entirely removed the corner effect.

We developed the calibration software by reusing parts of the MVisio for CAVE engine, thus creating again a server-client architecture. To simplify and accelerate the calibration procedure, a user just has to run this software and access the different grids and control points by using a wireless joystick. This way, the four walls can be calibrated at the same time by one user from within the CAVE, instead of having to login on every CAVE computer separately and adjust the grids with a mouse, one by one.

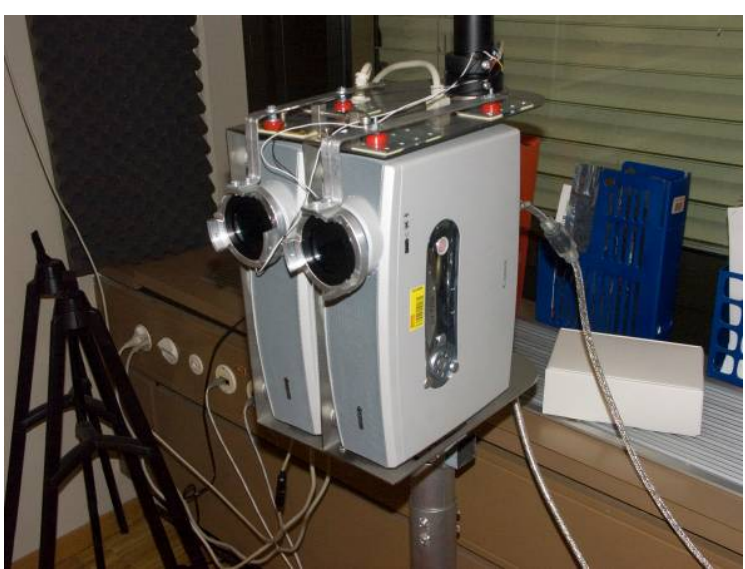

Figure 4: Dual-projector assembly with shutters.

This calibration solution also solved another problem. Our projectors have a maximal resolution of $1024 \times 768$ pixels. In order to cover the side wall according to the space available, we had to place them vertically, thus having different pixel resolutions between the main and floor screens (768 pixels vertically) and the right and left ones (1024 pixels). Moreover, pixel sizes on the CAVE walls are not the same on each wall, because of the different distances between the projectors and the displays. Antialiasing combined with an accurate match of the render-to shapes made this problem almost not noticeable. By using these techniques, we managed to achieve a continuous image on the different walls which creates a great immersion. To improve it a step further, in the next part we describe how we added depth information to the virtual scene through stereoscopic rendering.

\subsection{Stereographic rendering}

Stereographic rendering requires two images to be generated and displayed at the same time, one computed for the right and one for the left eye. Top-level solutions use a high refresh rate CRT projector fea- 
turing up to $120 \mathrm{~Hz}$ as refresh frequency. Through the use of shutter glasses, such systems can display a maximum of 60 images per second per eye. The video projector has to be driven by a professional graphic card which includes a synchronization output for the shutter glasses. This output changes the polarity of the shutter glasses according to the vertical synchronization of the display. Professional graphic cards and high quality CRT projectors are very expensive and require specific periodical cares to correct convergence.

We used a different approach in our system by adopting two standard LCD projectors (one per eye) with a built-in refresh rate of about $60 \mathrm{~Hz}$ (through a DVI cable). To achieve proper superposition of the two rendered images we built home made supports for the projectors. These supports allow fine adjustment of the position and orientation of the beamers.

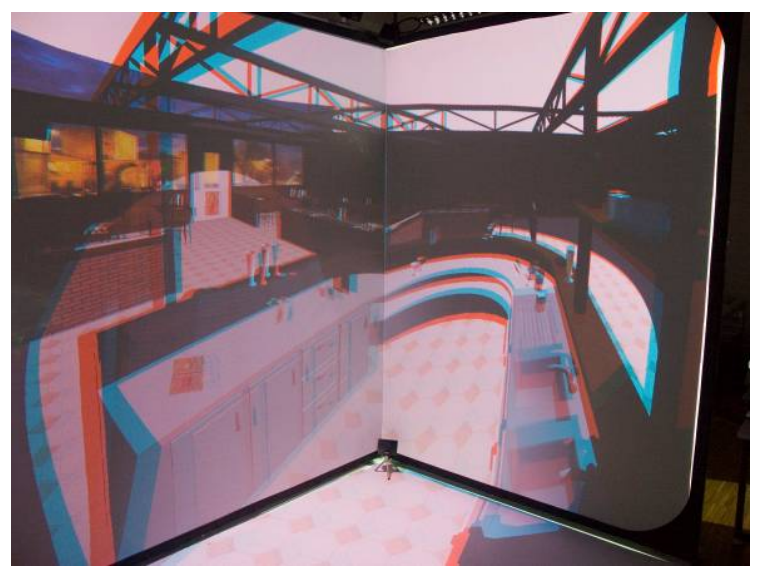

Figure 5: Blue \& red stereo in the CAVE.

The idea is to let the two LCD beamers continuously showing the images and synchronizing left and right eyes by putting ferroelectric shutters in front of the LCD lenses. This way, user shutter glasses synchronized with the ferroelectric shutters in front of the projectors allow a fixed number of images per second per eye, independently from the refresh rate of the display devices. In our configuration we used a switching between left and right eyes at $70 \mathrm{~Hz}$ which is the limit of the shutter glasses we adopted.

We also implemented an old-style blue \& red glasses stereographic system to be used during public demonstrations when a large number of visitors access the CAVE at the same time, because of the low amount and fragility of the shutter glasses. Finally, blue \& red glasses can also be used for stereographic testing on the virtual CAVE (see 4.6) on a single computer.

Stereographic rendering is improving immersion by adding depth information to the surrounding image. But while the user is moving, the perspective referential should be accordingly displaced to avoid break in presence. The next part will present our solutions to correct this effect by tracking the user head.

\subsection{Head tracking, environment walk- through}

Head tracking is a key feature in a CAVE system required to correctly generate the illusion of a surrounding space around a specific user. Headtracking is used to determine the position of the user within the CAVE physical space, in order to know his/her distance from the walls and compute a correct projection.

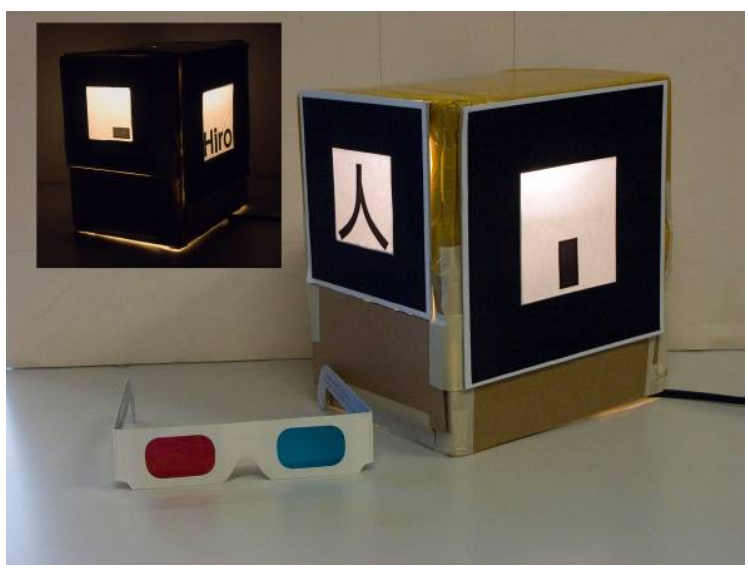

Figure 6: the cheapest solution: blue $\&$ red glasses with a head-wearable lantern. Detail picture on the left shows the lantern in the darkness.

For testing purposes we implemented three different methods to track the user's head position. The first method we studied was to use a camera and a vision based algorithm to track markers located on the user's head. The open source library ARtoolkit provided all the necessary functions to treat the video information in real time and extract $3 \mathrm{D}$ position and orientation of the predefined markers. We used a standard Panasonic video camera for the acquisition. The main issue in the setup is the weakness of the vision based algorithms to luminosity changes. At first we had very poor results due to the main source of illumination on the markers coming from the image displayed on the CAVE itself. This illumination of the markers was changing frequently and degrades the tracking accuracy. To solve this problem, a light bulb has been placed inside a small cube covered with markers fixed on the user head, creating some kind of head-worn lantern with semitransparent markers on the sides, to keep their luminosity constant and independent from the brightness of the images rendered on the CAVE walls. This 
system provided low tracking resolution at low refresh rate. With proper calibration and filtering it provides nice results to adjust to small user movements.

As second tracking method we used magnetic trackers. These sensors are composed by three inductances which measure the magnetic field induced by a referential field generator. The whole system is built by Ascension technologies under the name Motion Star system. This system provides 6 degree of freedom tracking with a really high resolution for the orientation and around $1 \mathrm{~cm}$ of accuracy for the positioning. The main advantage of this system is the high refresh rate with a correct accuracy.

We recently acquired an optical tracking system with active markers using multiple viewpoints. This system is composed by a set of linear video cameras which keep track of different LEDs which blinks according to their identifier. A complete dedicated hardware is in charge of the acquisition from multiple view points and computing of the positions of the markers. This system provides great accuracy around a few millimetres in positioning, which gives perfect results for our kind of applications.

To simplify calibration of sensors within the CAVE space and generalize the conversion between the values returned and the CAVE coordinates, we developed a simply and fast method using reference images projected on the CAVE walls and three laser pointers. We built a three orthogonal axis rigid support to put three laser pointers onto with a place where to lock the sensors on its origin. By aligning the three laser dots on a reference grid displayed on the walls and the floor, it is possible to easily match the reference points for converting sensor spatial coordinates to CAVE coordinates.

\subsection{Virtual CAVE}

Developing for a CAVE environment often requires the programmers to be near the hardware and to test directly on the device. This solution isn't always practical, mainly when different concurrent projects share the same CAVE at the same time.

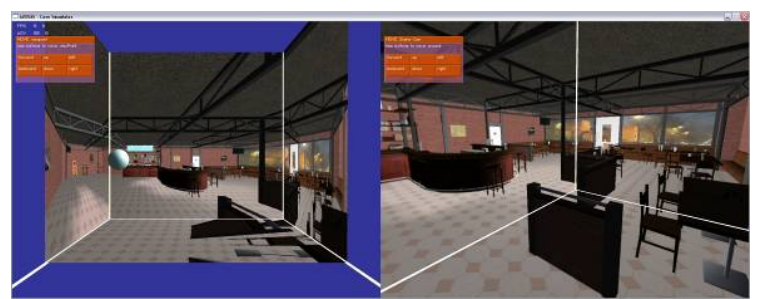

Figure 7: Bar scene displayed in the CAVE simulator. On the left, the white sphere represents the user's head, on the right images as viewed from the user's position.
To make development of CAVE-based applications independent from the physical device, we developed a software CAVE simulator, running exactly like the real one but in a system window. The virtual CAVE also features blue \& red stereographic rendering, thus enabling a very accurate reproduction of the stereographic images that will be generated on the real device.

Virtual Cave aims at providing an easy testing tool for developers, for draft evaluation of the system. In order to provide a global fair evaluation, the next part will discuss the test and results of our system.

\section{EVALUATION}

We based this evaluation on some concrete applications we developed in the laboratory with our framework as case of study.

\subsection{Applications}

The first application is a videogame developed as semester projects by students in our laboratory. The goal of this project was to create software accessing all the functionalities offered by our platform. Students developed a first person shooting game where the user holds a position tracked toy gun as input device for aiming at enemies. This game has also been used as demo to show to guests and visitors coming in our lab.

The second application used our framework to display virtual humans animated in real-time through an inverse kinematics algorithm. Virtual humans reacted by imitating the postures of a user staying within the CAVE and wearing led-based optical markers.

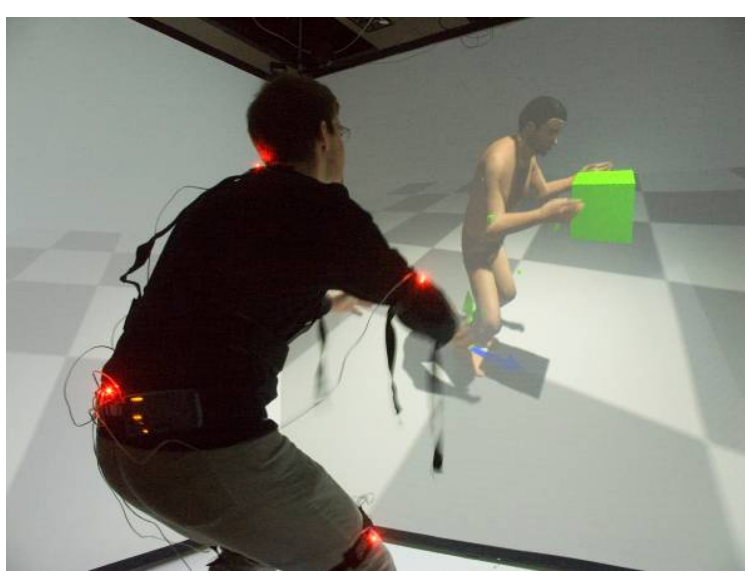

Figure 8: virtual human miming the movement effectuated by the user through an IK algorithm. 


\subsection{User feedbacks}

The first application gave us a large amount of feedback from a very heterogeneous amount of users, ranging from primary school students to Computer Graphics specialists.

Every user has been so far surprised by the quality both of the rendering and displaying of the images and found the illusion of being surrounded by a virtual environment convincing. Some of them complained about the black border around the cinema screen and cutting continuity between the wall displays and the floor (see figures with CAVE images). We are trying to raise the floor panel in order to avoid this break in presence.

Users disliked long sessions with blue \& red glasses because of the strong ghost effect showing part of the right image on the left eye and vice versa.

\subsection{Discussion}

Our system showed a good global luminosity even when used with ferroelectric shutters and shutter glasses. Despite the significant amount of luminosity lost through the shutters and the retro projection, the final images feature all the details, even if slightly dimmed. User tests also reported that bloom lighting improved the sensation of luminosity, without changing anything at the hardware level.

Our calibration software (either for walls and sensors) offered a robust workaround to manage the irregularities of the screen display and the glitch between projected images. Our approach also showed is usefulness to quickly correct some mismatching that may occurs due to dilatation of the support according to CAVE room temperature variations. Very small modifications on the beamer supports may rapidly become a few pixels misalignment when projected on the display: our software allowed a user to correct them in a couple of minutes.

We used relatively small ferroelectric shutters which caused a significant drawback: overheating. When closed, shutters block a high amount of light which raises their temperature. When the CAVE is active for long-time sessions (more than one hour, as we noticed during public demos with the first application), special cares have to be taken in the account, like some kind of screen-saver or heating monitoring. Alternatively, blue \& red glasses can be used and the shutters removed. We mounted them on a magnetic support to rapidly switch between the two modalities (see fig. 4: red disks are magnets).

All the different tracking systems we tested are suitable for application but each one with some specific limitations. ARtoolkit markers offer the cheapest solution but the lack of dedicated hardware, the sensitivity to the variable illumination, limitation of the field of view and occlusions due to the use of a single camera make the resultant tracking a bit unstable and a refresh rate around a few $\mathrm{Hz}$. With proper software filtering this solution offers acceptable results in a restricted area.

The magnetic tracking measurement is very accurate in orientation but the positioning is fairly poor in our case. In fact these types of sensors use the attenuation of the magnetic field to measure the distance to the referential generator. The main problem is coming from the fact that magnetic fields are perturbed by metallic masses. Since the CAVE frame responsible of the screen support is made of metal, the usable area of this system is limited as a meter cube around head's high in the center of the CAVE with an accuracy of about a couple of centimetres.

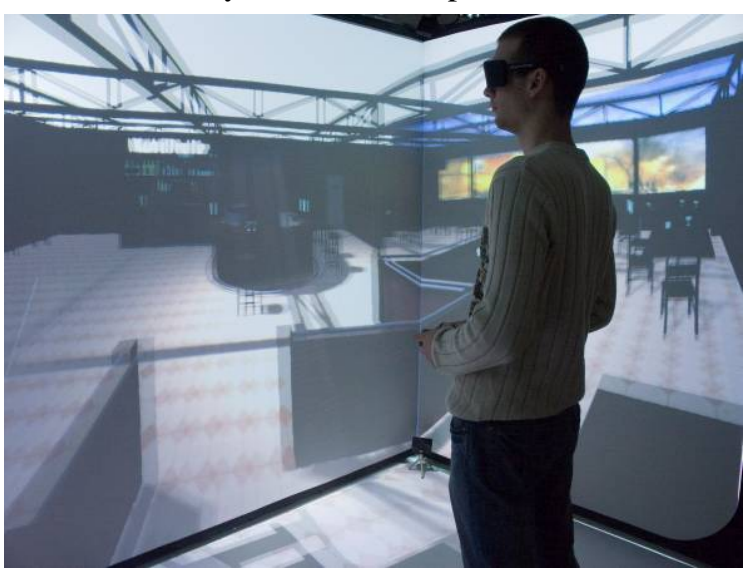

Figure 9: stereographic rendering.

The multiple camera optical tracking system offers an amazing accuracy around a few millimetres after a proper calibration and a fair range of effectiveness. The main disadvantage is that it requires camera located on the floor corner and multiple active markers on the user head but nothing compared to the bulky ARToolKit lantern.

On the graphic engine side, generating two images (left and right) per frame on a single PC may seem computational expensive, but it wasn't the case. Modern graphic cards can process geometry extremely quickly and thanks to extensions like the frame buffer object, render to texture come almost for free. Moreover, we were limited by the relatively low projector resolution of $1024 \times 768$, thus sparing some filling rate to be used for anti-aliasing or image post-processing (Gaussian filtering, bloom lighting). The most expensive feature we implemented is softshadowing. We used a shadow map-based algorithm which requires an additional pass for every light source, thus making two additional passes when in stereographic mode. We also used very high resolution light maps (2048x2048 texels) to improve video 
quality. All these graphic improvements may stress the hardware and rapidly killing the framerate. Despite of this, the bar scene used in our test had about 15.000 triangles, high resolution textures and still ran between around $25 \mathrm{fps}$ in stereographic mode, with one light source casting soft shadows and bloom lighting activated. It is important to mention that our engine is entirely dynamic, so that you can completely change the scene at run time, but nullifies different optimizations that could be used to significantly speed up the rendering procedure (contrarily to most gaming engines which often pregenerate an optimized environment but static). Our engine also features an almost direct use of models exported from 3D Studio MAX though a proprietary plug-in, without requiring any special care or conversion: other CAVE solutions based on videogames graphic engines, even if faster, put usually more constraints about that.

\section{CONCLUSION}

In this paper we exposed the different approaches we applied during the development of a high quality stereographic CAVE from the scratch. We showed how it is possible to build a very good system without requiring professional equipment, thanks to the high standard of quality of recent common market level products and a bit of practical sense. Software side, we created our framework by readapting portions of code from an existing graphic engine, rapidly getting a robust, performing and complete solution in a relatively short time.

Our system has shown its versatility and quality on real applications and user tests, and also goes in the direction evoked by Hibbard (Hibbard, 2000) about three major constraints limiting fruition of VR immersive devices: our framework offers a relatively cheap solution, fast sensor response and easiness of use on real contexts.

In the next phase we will refine our system and use it in a wider range of both scientific and business-oriented projects, in order to extend the fruition of this framework to other areas requiring immersive content visualization.

\section{REFERENCES}

Sutcliffe, A., Gault, B., Fernando, T., and Tan, K. 2006. Investigating interaction in CAVE virtual environments. ACM Trans. Comput.-Hum. Interact. 13, 2 (Jun. 2006), 235-267
Tyndiuk, F., Thomas, G., Lespinet-Najib, V., and Schlick, C. 2005. Cognitive comparison of 3D interaction in front of large vs. small displays. In Proceedings of the ACM Symposium on Virtual Reality Software and Technology, Monterey, CA, USA (Nov. 2005).

Buxton, B. and Fitzmaurice, G. W. 1998. HMDs, Caves \& chameleon: a human-centric analysis of interaction in virtual space. SIGGRAPH Comput. Graph. 32, 4 (Nov. 1998), 69-74.

Czernuszenko, M., Pape, D., Sandin, D., DeFanti, T., Dawe, G. L., and Brown, M. D. 1997. The ImmersaDesk and Infinity Wall projection-based virtual reality displays. SIGGRAPH Comput. Graph. 31, 2 (May. 1997), 46-49.

Cruz-Neira, C., Sandin, D.J., DeFanti, T.A., Kenyon, R., and Hart, J.C. 1992. The CAVE, Audio Visual Experience Automatic Virtual Environment. Communications of the ACM, June 1992, pp. 64-72.

Sauter, P. M. 2003. $\mathrm{VR}_{2} \mathrm{Go}^{\mathrm{TM}}$ : a new method for virtual reality development. SIGGRAPH Comput. Graph. 37, 1 (Feb. 2003), 19-24.

Gross, M., Würmlin, S., Naef, M., Lamboray, E., Spagno, C., Kunz, A., Koller-Meier, E., Svoboda, T., Van Gool, L., Lang, S., Strehlke, K., Moere, A. V., and Staadt, O. 2003. Blue-c: a spatially immersive display and 3D video portal for telepresence. ACM Trans. Graph. 22, 3 (Jul. 2003), 819-827.

Jacobson, J. 2003. Using “CAVEUT” to Build Immersive Displays With the Unreal Tournament Engine and a PC Cluster, http://citeseer.ist.psu.edu/639123.html

Jacobson, J., Hwang, Z. 2002. Unreal Tournament for Immersive Interactive Theater. Communications of the. ACM. 45, 1 (2002), 39-42.

Peternier, A., Thalmann, D., Vexo, F., Mental Vision: a computer graphics teaching platform, In Lecture Notes in Computer Science, Springer-Verlag Berlin, 2006

Jae Phil Koo, Sang Chul Ahn, Hyoung-Gon Kim, Ig-Jae Kim, "Active IR Stereo vision based tracking system for immersive displays," ACCV 2004, Jan. 2004.

M. Ghazisaedy, D. Adamczyk, D.J. Sandin, R.V. Kenyon, T.A. DeFanti, "Ultrasonic calibration of a magnetic tracker in a virtual reality space," vrais, p. 179, Virtual Reality Annual International Symposium (VRAIS'95), 1995.

Hibbard, B. 2000. Visualization spaces. SIGGRAPH Comput. Graph. 34, 4 (Nov. 2000), 8-10. 